\title{
Maspin expression and melanoma progression: a matter of sub-cellular localization
}

\author{
Chiara Martinoli ${ }^{1}$, Sara Gandini ${ }^{2}$, Chiara Luise ${ }^{3}$, Giovanni Mazzarol ${ }^{3}$, \\ Stefano Confalonieri ${ }^{3}$, Pier Giuseppe Pelicci ${ }^{4}$, Alessandro Testori ${ }^{5}$ and \\ Pier Francesco Ferrucci ${ }^{1}$
}

\begin{abstract}
${ }^{1}$ Medical Oncology of Melanoma Unit, Division of Medical Oncology, European Institute of Oncology, Milan, Italy; ${ }^{2}$ Division of Statistics and Epidemiology, European Institute of Oncology, Milan, Italy; ${ }^{3}$ Molecular Medicine for Care Program, European Institute of Oncology, Milan, Italy; ${ }^{4}$ Department of Experimental Oncology, European Institute of Oncology, Milan, Italy and ${ }^{5}$ Division of Melanoma and Soft Tissue Sarcomas, European Institute of Oncology, Milan, Italy
\end{abstract}

\begin{abstract}
Maspin, a member of the serpin family of protease inhibitors, is involved in key processes of cancer progression. Its biological activity seems to be cancer and compartment specific, with the protein acting either as a suppressor or as a tumor promoter in different cancer types. Characterization of maspin expression and its sub-cellular localization in melanoma is missing, hence, we aim to investigate its possible association with melanoma prognostic factors and disease progression. Nuclear and cytoplasmic maspin expression were evaluated on 60 nevi, 152 primary lesions, and 106 melanoma metastases using tissue microarrays and immunohistochemistry. The association between maspin immunoreactivity and patient's clinic-pathological features was evaluated. Multivariate logistic models and survival analyses were performed for maspin expression in primary melanomas. Nuclear maspin was detected in $8 \%$ nevi, $49 \%$ primary melanomas, and $28 \%$ metastases, whereas cytoplasmic maspin in $12 \%$ nevi, $18 \%$ primary lesions, and $9 \%$ metastases. In univariate analysis, nuclear maspin expression in primary melanomas was significantly associated with melanoma prognostic factors (nodular histotype, tumor thickness, mitotic rate, and ulceration) and disease stage, whereas cytoplasmic maspin was observed at higher frequency in thin superficial spreading melanomas, without mitosis. In multivariate analysis, nuclear maspin remained significantly associated with risk of developing a tumor prone to disease progression and, accordingly, with significantly shorter disease-free and overall survival. In this study, maspin was expressed at highest frequency in primary lesions and when expressed in the nuclei, was significantly associated with poor prognostic markers, melanoma recurrence, and worse survival. The present study suggests a tumor-suppressive effect of cytoplasmic maspin and a tumor-promoting effect of nuclear maspin, which open the discussion on its potential use in cancer therapy.

Modern Pathology (2014) 27, 412-419; doi:10.1038/modpathol.2013.157; published online 13 September 2013
\end{abstract}

Keywords: biomarker; maspin; melanoma; tissue microarrays

Melanoma is a malignant neoplasm that originates from melanocytes and it is characterized by frequent local recurrence, early metastasis, and refractoriness to chemotherapy and biological treatment. ${ }^{1}$ With early diagnosis and adequate surgical treatment of primary tumors (Stage I patients), the 5-year

Correspondence: Dr PF Ferrucci, MD, Medical Oncology of Melanoma Unit, Division of Medical Oncology, European Institute of Oncology, Milan 20141, Italy.

E-mail: pier.ferrucci@ieo.it

Received 22 April 2013; revised 5 July 2013; accepted 7 July 2013; published online 13 September 2013 survival rate is $>95 \% .^{2}$ However, once melanoma has metastasized to distant sites, life expectancy dramatically declines, with 1 -year survival rate of $33 \%$ for patients with Stage IV metastatic disease. ${ }^{2}$ Primary tumor thickness, ulceration, and mitotic rate, together with the presence of lymph node and systemic metastases, are the histopathologic criteria used for melanoma staging to predict disease outcome in terms of life expectancy and survival rates. ${ }^{2}$ However, a significant minority of melanomas contravenes these conventional parameters and displays unpredictable clinical behavior. Furthermore, lack of effective therapies for metastatic 
melanoma patients has only partially been overcome with introduction of anti-CTLA4 antibodies and BRAF inhibitors.

Maspin is a $42-\mathrm{kDa}$ protein encoded by the gene SERPINB5 that was originally identified in human mammary epithelial cells. ${ }^{3}$ Downregulation of maspin expression was observed in breast, prostate, bladder, oral, and lung cancer, and it was found to be associated with tumor progression and poor prognosis. ${ }^{4-9}$ Experimental data showing maspin involvement in all key biological processes contributing to cancer progression provided mechanistic support for the original definition of maspin as a tumor suppressor. In fact, maspin has been found to be able to inhibit tumor cell motility, invasion, and metastasis, ${ }^{10-13}$ to act as an inhibitor of angiogenesis ${ }^{14}$ and as a pro-apoptotic molecule in breast and prostate cancer cells ${ }^{15-18}$ and in endothelial cells. ${ }^{19}$

On the other hand, several clinical studies have questioned the tumor-suppressive activity of maspin, in colorectal, ${ }^{20}$ pancreatic, ${ }^{21}$ ovarian, ${ }^{22}$ and even in breast cancer. ${ }^{5,23}$ In this regards, it is important to underline that maspin can present as a nuclear, a cytoplasmic, a secreted, as well as a cell surfaceassociated protein..$^{24,25}$ Sub-cellular localization and tissue context might account for specific biological functions and differences in prognostic significance, as shown for breast ${ }^{23}$ and colorectal cancer. ${ }^{26}$

In melanoma, in vitro and in vivo experiments supported the idea that loss of maspin might have a role in disease progression, invasion, and metastatic dissemination, thereby suggesting that maspin might act as a tumor suppressor. ${ }^{27,28}$ Clinically, maspin expression in nevi and melanomas has been analyzed by four different groups, with different results. Wada et al found maspin in 38\% thin primary melanomas but not in nevi nor in thick primaries. ${ }^{29}$ The staining was reported to be cytoplasmic. The group of Vereecken observed a mixed nuclear-cytoplasmic maspin expression in 31\% nevi, $100 \%$ thin and $92 \%$ thick primary melanomas, and $70 \%$ metastases. ${ }^{30}$ By using a tissue microarray, Denk et al found maspin in only 1 out of 253 analyzed samples. ${ }^{27}$ Finally, Chua et al observed maspin in $100 \%$ nevi, $78 \%$ radial growth phase and $46 \%$ vertical growth phase primary melanomas. In this case, maspin staining was reported as exclusively nuclear, and, interestingly, showed an inverse correlation with the degree of angiogenesis. ${ }^{31}$ These studies suggest that also in melanoma maspin can present in different sub-cellular compartments, as observed in other cancer types, although this point was not specifically addressed.

Recent publications showing the importance of protein location for its biological activity prompted us to specifically investigate maspin expression and localization in a large set of nevi, primary melanomas, and metastases.

The most compelling data regarding the clinical significance of maspin in cancer progression and metastasis emerges from cancer patient survival studies. Although the original observations pointed to the association of reduced maspin expression with cancer progression, ensuing studies have revealed this correlation to be far more complex than originally concluded. Inevitably, further studies are required to shed further light on the usefulness of maspin as a prognostic marker to assist in tailored therapeutic approaches for specific cancers. We will evaluate the association of maspin with melanoma disease progression and the potential use of maspin in melanoma therapy, looking at maspin expression in different sub-cellular compartments.

\section{Materials and methods}

\section{Tissue Microarray Generation and Analysis}

For this study, three melanoma-specific tissue microarrays (TMA1-3) were used. TMA1 was composed of 17 nevi, 27 primaries, and 50 metastases; TMA2 contained 43 nevi and 63 metastases; TMA3 was composed of 133 primary tumors. There was a partial overlap among tissue microarrays, with eight primaries being represented on TMA1 and TMA3, and seven metastases on TMA1 and TMA2. Therefore, globally tissue microarrays allowed analysis of 60 benign lesions (nevi), 152 primary tumors, and 106 melanoma metastases. Human specimens were arrayed from formalin-fixed paraffin-embedded tissues (donor blocks) of normal and malignant origin of patients who underwent surgery at European Institute of Oncology between 1995 and 2008. At least two representative areas (cores of $0.6 \mathrm{~mm}$ diameter) were arrayed from each donor block. For primary melanomas included in TMA3, up to six cores from each sample were arrayed.

Two-micrometer sections of each tissue microarray were cut, mounted on glass slides and processed for immunohistochemistry. After dewaxing in histolemon, sections were rehydrated in graded alcohol solutions and incubated in citrate buffer $\mathrm{pH} \quad 6$ for $50 \mathrm{~min}$ at $95{ }^{\circ} \mathrm{C}$ to perform antigen unmasking. After blocking, mouse anti-human maspin (BD Pharmingen, clone G167-70) was applied at $1.25 \mu \mathrm{g} / \mathrm{ml}$ for $2 \mathrm{~h}$, followed by LSAB + System Alkaline Phoshatase (Dako). After being stained 15 min with Vulcan Fast Red chromogen, sections were counterstained with hematoxylin, dehydrated, and mounted with Eukitt.

Maspin immunoreactivity was evaluated by two pathologists in a blinded manner. Each core included in the tissue microarray was assigned a score depending on maspin staining intensity (sample staining are shown in Figure 1), ranging from 0 to 3 (score 0: null; 1: weak; 2: moderate; 3: strong immunoreactivity). The mean immunoreactivity (immunoreactive score) was calculated for each sample considering available cores derived from 

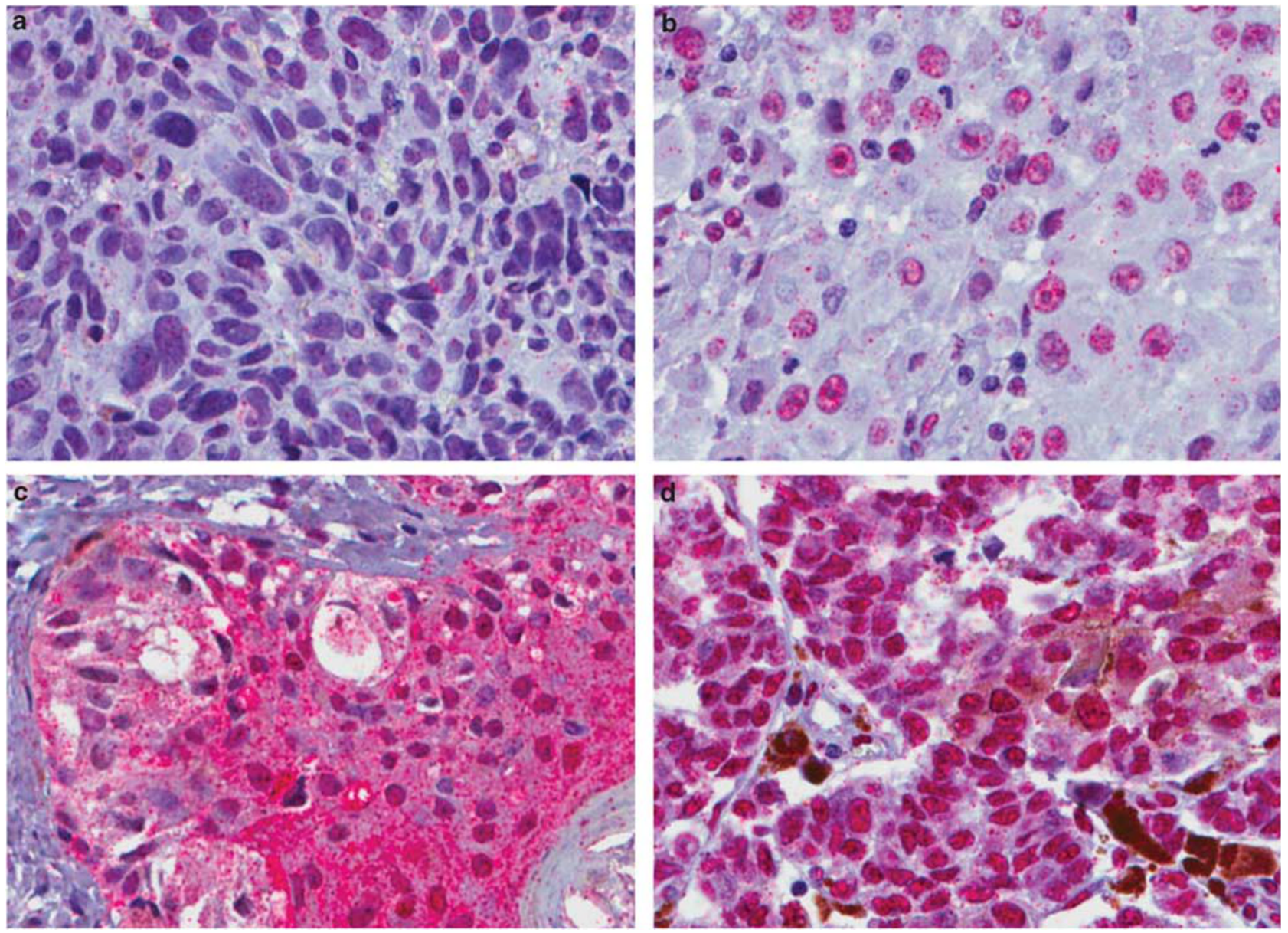

Figure 1 Maspin expression in melanoma samples. (a) A tissue core showing no maspin expression. (b) A tissue core with nuclear but not cytoplasmic maspin. (c) A tissue core with cytoplasmic but not nuclear maspin. (d) A tissue core with both nuclear and cytoplasmic maspin.

different tumor areas. Samples were considered positive for maspin expression if immunoreactive score was at least 1 , and nuclear and cytoplasmic staining were recorded separately.

\section{Statistical Methods}

Frequencies of positive maspin staining for nuclei and cytoplasms of melanoma cells are presented for each categories of known melanoma prognostic factors. Chi-squared or Fisher's exact tests were used to analyze the association between categorical variables. Risk analyses were conducted using logistic regression, and odds ratios, adjusted for significant confounding factors, were calculated in order to evaluate the association between maspin and prognostic factors.

Time to death and time to recurrence were defined as the time from surgery until the event of interest. All patients alive or free of disease at last follow-up date were considered right censored. Disease-free and overall survival were estimated by the KaplanMeier method (excluding stage IV melanoma). The log-rank tests were used to compare survival time between groups. A Cox proportional hazards model was used to evaluate the association of maspin expression with survival, adjusting for significant confounding factors such as age and sex. The analyses were not carried out adjusting for Breslow thickness, invasion, and nodular involvement because these prognostic factors cannot be considered as confounding variables. In fact, they can be considered intermediate steps in the casual path between maspin expression and relapse, as maspin has either a direct influence on cell invasion or it indirectly regulates target molecules that lead to reduced invasive potential.

All statistical tests were two-sided, and $P<0.05$ was considered statistically significant. The statistical analyses were performed with the Statistical Analysis System Version 9.2 (SAS Institute, Cary, NC, USA).

\section{Results}

\section{Maspin Expression and Melanoma Progression}

Maspin expression was evaluated in nevi, primary melanomas, and melanoma metastases by 
immunohistochemistry using tissue microarray technology. Maspin staining was quantified as described in Materials and methods, and recorded separately for nuclei and cytoplasms of melanoma cells (sample stainings are shown in Figure 1).

Nevi. Sixty nevi were included in the tissue microarrays, and analyzed for maspin expression (Table 1). The tissue microarrays included 27 benign and 33 dysplastic nevi. Nuclear maspin was present in five $(8 \%)$ pigmented lesions, whereas cytoplasmic maspin was observed in seven $(12 \%)$ nevi. Among these, only one sample stained positive both in the nuclear and cytoplasmic compartment. No significant differences as for maspin expression were observed between benign and dysplastic nevi.

Primary Melanomas. One hundred fifty-two primary melanomas were analyzed (Table 1). These lesions included 71 thin $(\leq 1.00 \mathrm{~mm})$ and 80 intermediate-thick (>1.01 mm) melanomas. For one sample, Breslow thickness was not available. Maspin was expressed in nuclei of $75(49 \%)$ samples, whereas cytoplasmic maspin was observed in 27 $(18 \%)$ lesions. A significant increased frequency of nuclear maspin expression was noted in intermediate-thick lesions $(P<0.0001)$, whereas cytoplasmic maspin-expressing lesions was twofold more frequent among thin lesions $(24 \%$ vs $12.5 \%$, not significant).

Melanoma Metastases. One hundred and six melanoma metastases were analyzed (Table 1). The tissue microarrays included 7 cutaneous, 90 lymph node, and 9 lung metastases. Nuclear maspin was present in $30(28 \%)$ samples, whereas cytoplasmic maspin

Table 1 Maspin expression in nevi, primary melanomas, and melanoma metastases

\begin{tabular}{|c|c|c|c|c|c|}
\hline \multirow[b]{3}{*}{ Tissue } & \multirow[b]{3}{*}{ Total } & \multicolumn{4}{|c|}{ Maspin-positive staining } \\
\hline & & \multicolumn{2}{|c|}{ Nuclear } & \multicolumn{2}{|c|}{ Cytoplasmic } \\
\hline & & $\mathrm{N}(\%)$ & P-value & $\mathrm{N}(\%)$ & $\mathrm{P}$-value \\
\hline $\begin{array}{l}\text { Nevi } \\
\text { Benign } \\
\text { Dysplastic }\end{array}$ & $\begin{array}{l}60 \\
27 \\
33\end{array}$ & $\begin{array}{r}5(8) \\
1(4) \\
4(12)\end{array}$ & 0.24 & $\begin{array}{c}7(12) \\
2(7) \\
5(15)\end{array}$ & 0.35 \\
\hline $\begin{array}{l}\text { Primaries } \\
\text { Thin } \\
\text { Intermediate-thick }\end{array}$ & $\begin{array}{r}152 \\
71 \\
80\end{array}$ & $\begin{array}{l}75(49) \\
21(30) \\
53(66)\end{array}$ & $<0.0001$ & $\begin{array}{l}27(18) \\
17(24) \\
10(13)\end{array}$ & 0.067 \\
\hline $\begin{array}{l}\text { Metastases } \\
\text { Cutaneous } \\
\text { Lymph node } \\
\text { Lung }\end{array}$ & $\begin{array}{r}106 \\
7 \\
90 \\
9\end{array}$ & $\begin{array}{r}30(28) \\
1(14) \\
26(29) \\
3(33)\end{array}$ & 0.67 & $\begin{array}{c}10(9) \\
0(0) \\
9(10) \\
1(11)\end{array}$ & 0.67 \\
\hline
\end{tabular}

Abbreviation: N: number of patients for whom immunoreactive score IRS $\geq 1$.

Significance was calculated in univariate analysis by $\chi^{2}$ test. was observed in $10(9 \%)$ tissues. No significant differences were observed in maspin expression among different sites of metastatization, likely due to the low number of non-nodal metastases.

Maspin Expression Peaks in Primary Melanomas. Taken together, these data show that the frequency of nuclear maspin-expressing lesions increased from nevi to thin melanomas $(P=0.002)$, peaked in thick primary tumors $(P<0.0001$ when compared with all other tissues), and dropped down in metastases. Cytoplasmic maspin expression was less frequent than nuclear expression, and displayed a modest peak in thin lesions $(P=0.008$ when compared with metastases).

\section{Maspin Expression in Primary Melanomas}

Data presented above suggest that maspin expression and distribution in primary lesions could be representative of subsequent disease progression. Therefore, we decided to investigate more deeply the association of maspin expression and primary melanoma looking at known prognostic factors. As we had only 27 patients with cytoplasmic maspin expression, the main analyses were carried by nuclear maspin, independently of cytoplasmic score, and by cytoplasmic maspin expression, independently of nuclear score. Finally, we performed subgroup analyses considering simultaneous maspin expression in both compartments for negative cytoplasmatic maspin patients, which is the group with the greatest sample size.

Associations with Prognostic Factors. Clinicopathological features of patients included in the tissue microarrays are listed in Table 2. The tissue microarrays included primary melanomas from 73 male and 79 female patients, with a median age of 55.6 years at surgery. The median follow-up was 69 months. Information on tumor histotype, thickness, mitotic rate, ulceration, regression, and lymph node involvement were available for most of the patients.

We found that nuclear maspin (Table 2) was more frequent in nodular lesions than in superficial spreading melanomas $(P<0.0001)$. Nuclear maspin expression was significantly associated to melanoma thickness $(P<0.0001)$, mitotic rate $(P<0.0001)$, and the presence of ulceration $(P=0.002)$, but not with regression $(P=0.09)$. Nuclear maspin expression seemed to assist melanoma deepening, increasing from $30 \%$ positivity of pT1 lesions to $90 \%$ positivity of pT4 lesions.

Cytoplasmic maspin was found in $18 \%$ samples (Table 2). Cytoplasmic maspin was observed more frequently in superficial spreading than in nodular melanomas $(P=0.01)$, and in lesion with mitotic rate $<1 / \mathrm{mm}^{2} \quad(P<0.01)$. Cytoplasmic maspin expression was higher in thin lesions and in nonulcerated lesions, although differences did not reach 
Table 2 Maspin expression in primary lesions and its association to clinico-pathological features of patients included in the study

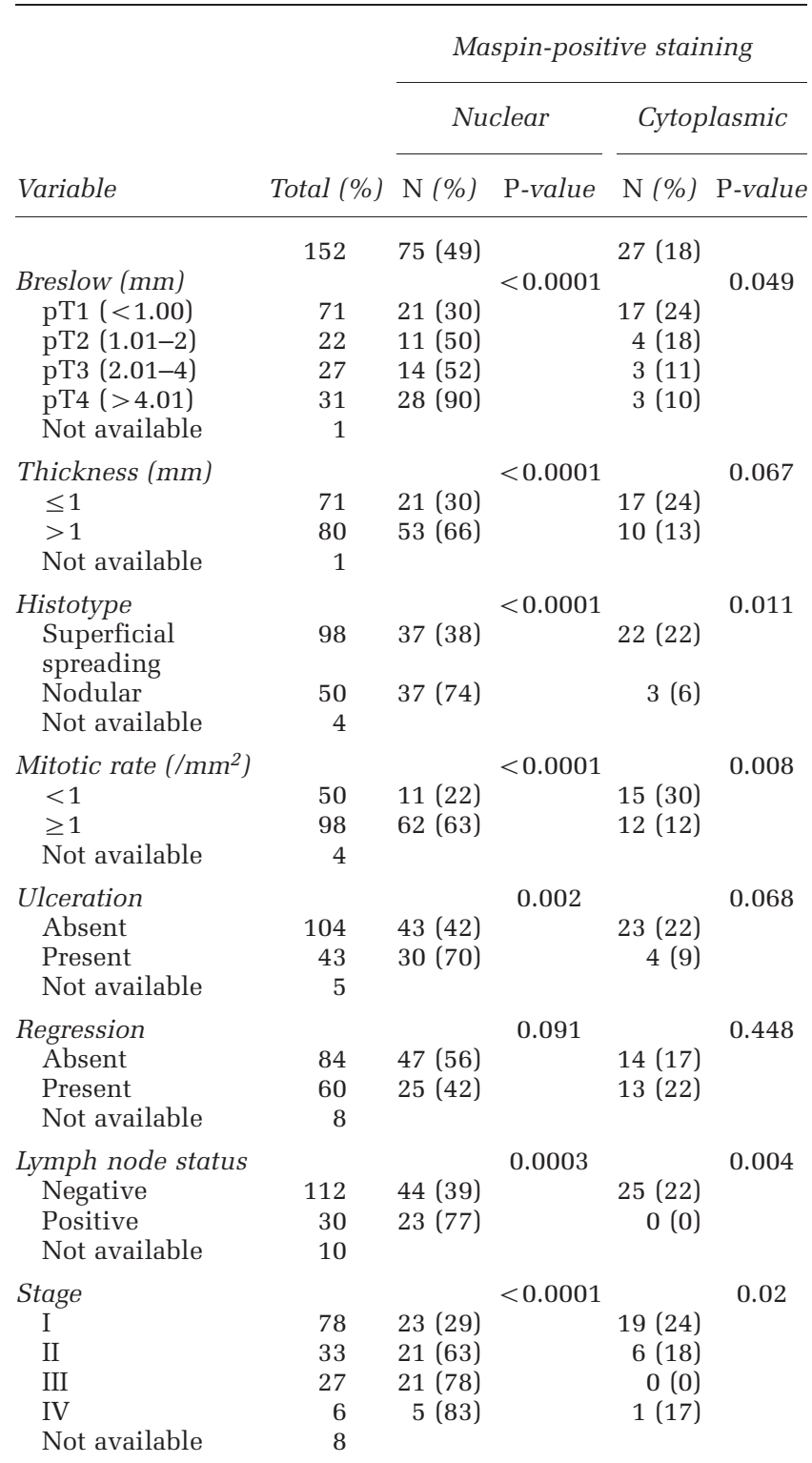

Abbreviation: $\mathrm{N}$ : number of patients for whom immune reactive score IRS $\geq 1$.

Significance was calculated in univariate analysis by $\chi^{2}$ or Fisher's exact test for sparse data and Mantel-Haenszel $\chi^{2}$ test to assess the trend.

statistical significance ( $P=0.07$ for both). Although only few samples stained positive for maspin in the cytoplasmic compartment, loss of cytoplasmic maspin seemed to assist melanoma deepening, with twofold higher frequency of pT1 than pT4-positive lesions.

Notably, maspin expression in primary lesions was also significantly associated to patient clinical stage (Table 2), with highest frequency of cytoplasmic positivity in stage I patients, and highest frequency of nuclear positivity in stage IV patients ( $P=0.02$ and $P<0.0001$, respectively). Accordingly, when we analyzed the association between maspin expression and lymph node involvement, we found that nuclear maspin was positively associated $(P=0.0003)$ to the presence of metastatic nodes, whereas none of patients displaying cytoplasmic maspin in their primary lesions had metastatic nodes $(P=0.004)$ at time of diagnosis (Table 2$)$.

In multivariate analyses, results are confirmed: nuclear maspin expression was highly significantly associated with well-known prognostic factors (Breslow $>1.00 \mathrm{~mm}$, the presence of ulceration and mitoses, lymph node metastases), as shown by odd ratios comparing patients with worse prognostic factors with patients with a lower degree of disease (Table 3). Metastatic lymph nodes and thick melanomas are five times more frequent with positive maspin $(P=0.001$ and $P<0.0001$, respectively). Positive maspin increased three times the probability of having ulcerated melanoma $(P=0.003)$ and six times the probability of having mitotic rates equal or greater than $1(<0.0001)$. A similar trend was observed for patients who, vice versa, did not express maspin in cytoplasmic compartment of primary melanoma cells, but the risk estimates reached significance $(P=0.014)$ only for mitotic rates (the association with lymph nodes could not be evaluated).

Survival Analyses. Disease-free and overall survival analyses confirmed associations found with prognostic factors. Kaplan-Meier survival curves for overall survival are shown in Figure 2 and Hazard ratios from Cox models adjusted for age in Table 3. We found that nuclear maspin-expressing patients had a significantly higher rate of recurrence and shorter overall survival than nuclear maspinnegative patients (Figure 2a; $P=0.02$ and $P=0.01$, respectively). Conversely, cytoplasmic maspin-positive patients displayed lower, although not statistically significant, recurrence and mortality when compared with negative patients (Figure $2 \mathrm{~b} ; P=0.15$ and $P=0.06$, respectively).

Analyses in Cytoplasmic Negative Patients. Characterization of maspin expression and tumor features revealed a sub-cellular compartment-dependent association to prognostic factors: nuclear and cytoplasmic maspin seemed to have opposite roles in melanoma cells, with cytoplasmic protein acting as a tumor suppressor, and nuclear maspin as a tumor promoter.

In order to confirm pure nuclear maspin tumor promoting effect, we analyzed the association of nuclear protein to prognostic factors in cytoplasmic negative patients. The limited number of cytoplasmic positive patients, did not allow us to perform the reciprocal analysis in nuclear negative patients.

In line with our first analysis, we observed (Figure 2c) a strong association of nuclear maspin to nodular histotype $(P=0.0001)$, thickness $(P<0.0001)$, mitotic rate $(P<0.0001)$, ulceration $(P<0.0001)$, and 
Table 3 Multivariate logistic and Cox regression models adjusted for age

\begin{tabular}{|c|c|c|c|c|c|}
\hline \multirow[b]{2}{*}{ Variable } & \multirow[b]{2}{*}{ Contrasts } & \multicolumn{2}{|c|}{ Nuclear: positive vs negative } & \multicolumn{2}{|c|}{ Cytoplasmic: negative vs positive } \\
\hline & & OR $(95 \% C I)$ & P-value & OR $(95 \% C I)$ & $\mathrm{P}$-value \\
\hline Thickness & $>1 \mathrm{~mm} \mathrm{vs} \leq 1 \mathrm{~mm}$ & $4.86(2.37,9.95)$ & $<0.0001$ & $2.06(0.86,4.95)$ & 0.106 \\
\hline Ulceration & Present vs absent & $3.16(1.47,6.77)$ & 0.003 & $2.69(0.87,8.33)$ & 0.087 \\
\hline Mitotic rate & $\geq 1 / \mathrm{mm}^{2}$ vs $<1 / \mathrm{mm}^{2}$ & $6.05(2.74,13.34)$ & $<0.0001$ & $2.93(1.24,6.94)$ & 0.014 \\
\hline \multirow[t]{2}{*}{ Lymph node } & Positive vs negative & $5.08(2.01,12.85)$ & 0.001 & NA & \\
\hline & $\mathrm{N}$ events $/ \mathrm{N}$ at risk & HR $(95 \% C I)$ & $\mathrm{P}$-value & $H R(95 \% C I)$ & $\mathrm{P}$-value \\
\hline Recurrence (DFS) & $63 / 152$ & $2.04(1.21,3.45)$ & 0.008 & $1.82(0.71,4.64)$ & 0.211 \\
\hline Death (OS) & $54 / 152$ & $2.09(1.19,3.65)$ & 0.010 & $2.65(0.95,7.38)$ & 0.062 \\
\hline
\end{tabular}

Abbreviations: CI: confidence interval; DFS: disease-free survival; HR: hazard ratio; OR: odd ratio; OS: overall survival.

ORs and HRs are adjusted for age.

a

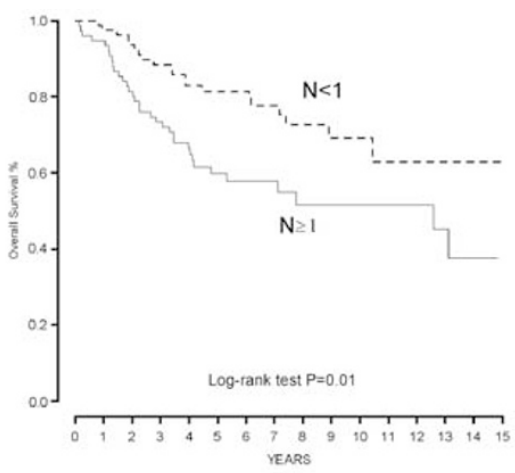

C

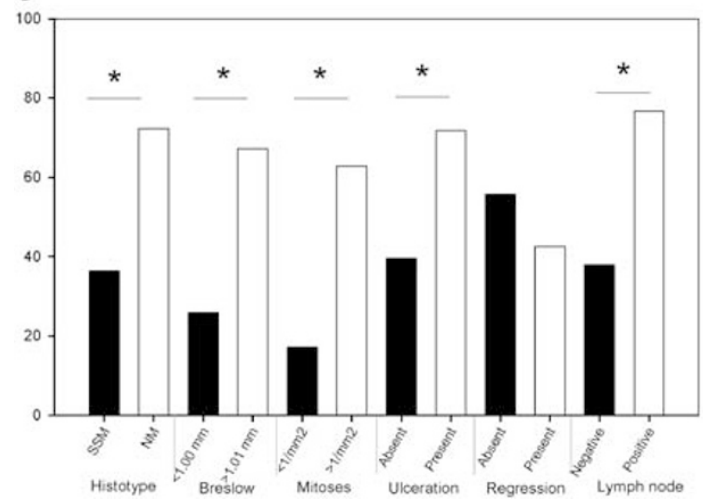

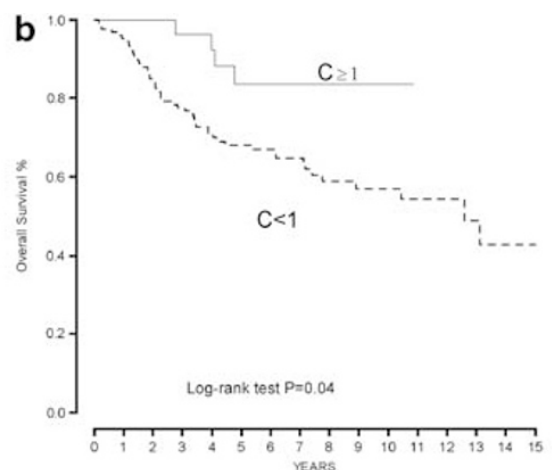

d

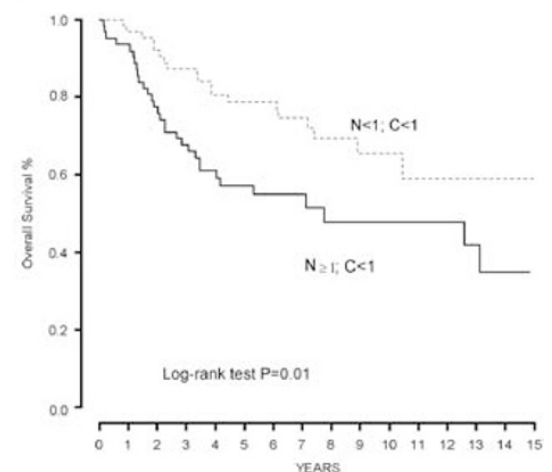

Figure 2 Correlation of maspin expression and patient's prognosis. Kaplan-Meier overall survival curves by nuclear (a) and cytoplasmic (b) maspin expression. (c) Association of nuclear maspin to primary tumor features in cytoplasmic-negative samples. Percentages of positive nuclear staining are shown; ${ }^{*} P<0.05$. (d) Kaplan-Meier overall survival curves in cytoplasmic negative patients. a, b, d: continuous line = positive staining; dotted line= negative staining. Log-rank test was used to compare curves.

lymph node involvement $(P=0.0002)$. In the absence of cytoplasmic protein, nuclear maspin is also significantly associated with disease-free and overall survival (Table 4 and Figure 2d).

\section{Discussion}

In the present study, we analyzed not only the frequency of maspin expression, but also its subcellular location, in a large set of melanocytic
Table 4 Multivariate Cox regression models for nuclear positive maspin among cytoplasmic negative patients

\begin{tabular}{lccc}
\hline & $\begin{array}{c}\text { N events/ } \\
\text { N at risk }\end{array}$ & $\begin{array}{c}\text { HR } \\
(95 \% \text { CI })\end{array}$ & P-values \\
\hline Recurrence (DFS) & $56 / 125$ & $2.16(1.24,3.79)$ & 0.007 \\
Death (OS) & $50 / 125$ & $2.05(1.15,3.66)$ & 0.015
\end{tabular}

Abbreviations: CI: confidence interval; DFS: disease-free survival; OS: overall survival; HR: hazard ratio.

HRs are adjusted for age. 
lesions, both benign (nevi) and malignant (primary melanomas and metastases). We found highest frequency of maspin expression in primary lesions: nuclear maspin was present in $8 \%$ nevi, $49 \%$ primary melanomas, and $28 \%$ metastases, whereas cytoplasmic maspin was observed in 12\% nevi, 18\% primaries, and 9\% metastases. Importantly, huge differences were noted when we analyzed the association of maspin distribution to primary tumor characteristics. In particular, nuclear maspin was significantly associated to poor prognostic markers: increased thickness, mitoses $\geq 1 / \mathrm{mm}^{2}$, the presence of ulceration and nodular histotype. Conversely, cytoplasmic maspin was present at higher frequency in thin superficial spreading melanomas, without mitoses and ulceration.

Furthermore, when we analyzed the association of maspin expression in primary tumors to lymph node involvement, we observed a significant association of nuclear maspin and lymph node positivity, whereas none of patients expressing maspin in cytoplasmic compartment of primary lesions had also metastatic nodes at the time of primary surgery. This clearly means that nuclear maspin expression is positively associated to tumor cell dissemination in regional lymph nodes, and the opposite for cytoplasmic maspin. In line with this observation, the opposite association between maspin expression/distribution and disease progression was confirmed when we looked at stages of disease.

On the other hand, maspin was originally identified as a tumor suppressor in breast and prostate cancer, as its expression is decreased and/or lost in neoplastic cells when compared with the normal counterpart. When maspin expression was analyzed in tissues of melanoma patients, the protein was found to be downregulated in tumors with a more aggressive phenotype, supporting the notion of its tumor-suppressive activity. However, a high degree of variability was reported by different groups as for the frequency of protein expression, with maspin positivity ranging from 0 to $94 \%$ in primary tumors, and from 0 to $100 \%$ in metastases. ${ }^{27,29-31}$ Interestingly, when maspin expression was examined in clinical samples from various cancers, with tumor cells being analyzed in the context of host, stromal, and environmental influences, conflicting results were obtained, and the tumor-suppressive activity of maspin has been questioned. From these studies, it is becoming evident that not only protein expression, but also its sub-cellular localization, and consequently its putative interactors and functions, may influence its association with disease outcome and patient prognosis. ${ }^{32}$ Further complexity in understanding maspin biology is given by the observation of opposite effects of maspin expression and localization in different cancer types. On the contrary to what observed in breast and prostate cancer, nuclear maspin was associated with increased angiogenesis in lung carcinoma and with poor survival in colorectal cancer. These studies point not to overlook the cell- and tissue-specific context in addition to protein expression and localization. Although studies performed on melanoma tissues did not specifically investigate the sub-cellular location of maspin, published data report the protein as nuclear, ${ }^{31}$ mixed nuclear and cytoplasmic, ${ }^{30}$ and purely cytoplasmic. ${ }^{29}$ To the best of our knowledge, this is the first study aimed at specifically examining the association of maspin expression and sub-cellular localization with disease progression in a large cohort of melanoma patients.

Our results suggest that in melanoma maspin may act as a tumor inducer when confined in the nucleus, and as a tumor suppressor when present in the cytoplasm. The molecular mechanism(s) through which maspin becomes nuclear localized, including interactors and environmental conditions that could favor its translocation, and the specific biological functions associated with nuclear localization are still largely unknown.

As maspin seems to participate to key biological processes of cancer progression, we believe that the mechanisms of maspin regulation and compartmentalization need to be further investigated. Our observations that nuclear maspin peaks in thick primary lesions compared with thin melanomas, and that is less abundant in the metastatic tissues, would suggest that the protein could be involved in early invasive steps of melanoma progression. In particular, we can speculate that this protein could be involved in regulating adhesion to extracellular matrix as well as influencing migratory potential, thus accounting for tumor cell propensity to metastatic spread. Other possible targets of maspin include the PTEN/AKT as well as the K-RAS/BRAF/ $M E K$ pathways, which are also controlling the molecular mechanisms responsible for melanoma cell proliferation and interaction with environment.

Maspin, which has a multitude of functions in cancer cell biology, being implicated in tumor cell adhesion, migration, invasiveness, apoptosis, and angiogenesis, would represent an ideal target for therapeutic intervention given the possibility of inhibiting melanoma cell metastatic potential while increasing their susceptibility to cytotoxic treatments.

In conclusion, determination of maspin immunoreactivity in primary melanomas would help in early assessment of tumor cell propensity to metastatic spread and could provide a target of intervention for limiting melanoma cell dissemination.

\section{Acknowledgements}

Chiara Martinoli is supported by Fondazione Grazia Focacci Onlus, Milan, Italy, a no profit Foundation. Part of the work have been supported by a grant from EORTC (European Organization for Research and Treatment of Cancer) Melanoma Group. 


\section{Disclosure/conflict of interest}

The authors declare no conflict of interest.

\section{References}

1 Mouawad R, Sebert M, Michels J, et al. Treatment for metastatic malignant melanoma: old drugs and new strategies. Crit Rev Oncol Hematol 2010;74:27-39.

2 Balch CM, Gershenwald JE, Soong SJ, et al. Final version of 2009 AJCC melanoma staging and classification. J Clin Oncol 2009;27:6199-6206.

3 Zou Z, Anisowicz A, Hendrix MJ, et al. Maspin, a serpin with tumor-suppressing activity in human mammary epithelial cells. Science 1994;263:526-529.

4 Maass N, Hojo T, Rosel F, et al. Down regulation of the tumor suppressor gene maspin in breast carcinoma is associated with a higher risk of distant metastasis. Clin Biochem 2001;34:303-307.

5 Umekita Y, Ohi Y, Sagara Y, et al. Expression of maspin predicts poor prognosis in breast-cancer patients. Int J Cancer 2002;100:452-455.

6 Machtens S, Serth J, Bokemeyer C, et al. Expression of the p53 and Maspin protein in primary prostate cancer: correlation with clinical features. Int J Cancer 2001;95: 337-342.

7 Beecken WD, Engl T, Engels K, et al. Clinical relevance of maspin expression in bladder cancer. World J Urol 2006;24:338-344.

8 Yasumatsu R, Nakashima T, Hirakawa N, et al. Maspin expression in stage I and II oral tongue squamous cell carcinoma. Head Neck 2001;23:962-966.

9 Takanami I, Abiko T, Koizumi S. Expression of maspin in non-small-cell lung cancer: correlation with clinical features. Clin Lung Cancer 2008;9:361-366.

10 Sheng S, Carey J, Seftor EA, et al. Maspin acts at the cell membrane to inhibit invasion and motility of mammary and prostatic cancer cells. Proc Natl Acad Sci USA 1996;93:11669-11674.

11 Seftor RE, Seftor EA, Sheng S, et al. Maspin suppresses the invasive phenotype of human breast carcinoma. Cancer Res 1998;58:5681-5685.

12 Shi HY, Zhang W, Liang R, et al. Blocking tumor growth, invasion, and metastasis by maspin in a syngeneic breast cancer model. Cancer Res 2001;61: 6945-6951.

13 Odero-Marah VA, Khalkhali-Ellis Z, Chunthapong J, et al. Maspin regulates different signaling pathways for motility and adhesion in aggressive breast cancer cells. Cancer Biol Ther 2003;2:398-403.

14 Zhang M, Volpert O, Shi YH, et al. Maspin is an angiogenesis inhibitor. Nat Med 2000;6:196-199.

15 Jiang N, Meng Y, Zhang S, et al. Maspin sensitizes breast carcinoma cells to induced apoptosis. Oncogene 2002;21:4089-4098.

16 Liu J, Yin S, Reddy N, et al. Bax mediates the apoptosis-sensitizing effect of maspin. Cancer Res 2004;64:1703-1711.
17 Zhang W, Shi HY, Zhang M. Maspin overexpression modulates tumor cell apoptosis through the regulation of Bcl-2 family proteins. BMC Cancer 2005;5:50.

18 Tahmatzopoulos A, Sheng S, Kyprianou N. Maspin sensitizes prostate cancer cells to doxazosin-induced apoptosis. Oncogene 2005;24:5375-5383.

19 Li Z, Shi HY, Zhang M. Targeted expression of maspin in tumor vasculatures induces endothelial cell apoptosis. Oncogene 2005;24:2008-2019.

20 Bettstetter M, Woenckhaus M, Wild PJ, et al. Elevated nuclear maspin expression is associated with microsatellite instability and high tumour grade in colorectal cancer. J Pathol 2005;205:606-614.

21 Maass N, Hojo T, Ueding M, et al. Expression of the tumor suppressor gene Maspin in human pancreatic cancers. Clin Cancer Res 2001;7:812-817.

22 Sood AK, Fletcher MS, Gruman LM, et al. The paradoxical expression of maspin in ovarian carcinoma. Clin Cancer Res 2002;8:2924-2932.

23 Mohsin SK, Zhang M. Clark GM et al. Maspin expression in invasive breast cancer: association with other prognostic factors. J Pathol 2003;199:432-435.

24 Pemberton PA, Tipton AR, Pavloff N, et al. Maspin is an intracellular serpin that partitions into secretory vesicles and is present at the cell surface. J Histochem Cytochem 1997;45:1697-1706.

25 Khalkhali-Ellis Z, Hendrix MJ. Elucidating the function of secreted maspin: inhibiting cathepsin D-mediated matrix degradation. Cancer Res 2007;67: 3535-3539.

26 Markl B, Arnholdt HM, Jahnig H, et al. Shift from cytoplasmic to nuclear maspin expression correlates with shorter overall survival in node-negative colorectal cancer. Hum Pathol 2010;41:1024-1033.

27 Denk AE, Bettstetter M, Wild PJ, et al. Loss of maspin expression contributes to a more invasive potential in malignant melanoma. Pigment Cell Res 2007;20: 112-119.

28 Villares GJ, Zigler M, Dobroff AS, et al. Protease activated receptor-1 inhibits the Maspin tumor-suppressor gene to determine the melanoma metastatic phenotype. Proc Natl Acad Sci USA 2011;108: 626-631.

29 Wada K, Maesawa C, Akasaka T, et al. Aberrant expression of the maspin gene associated with epigenetic modification in melanoma cells. J Invest Dermatol 2004;122:805-811.

30 Vereecken P, Reynaert S, Lalmand MC, et al. Decreased immunoreactive maspin expression in intermediate thickness and thick primary melanoma lesions. J Int Med Res 2006;34:52-57.

31 Chua R, Setzer S, Govindarajan B, et al. Maspin expression, angiogenesis, prognostic parameters, and outcome in malignant melanoma. J Am Acad Dermatol 2009;60:758-766.

32 Goulet B, Chan G, Chambers AF, et al. An emerging role for the nuclear localization of maspin in the suppression of tumor progression and metastasis. Biochem Cell Biol 2012;90:22-38. 